\title{
Sozialpsychiatrie kann als eigenständiges Gebiet abgeschafft werden - Kontra
}

\author{
Social Psychiatry can be Disestablished as a Separate Field - Contra
}

Kontra

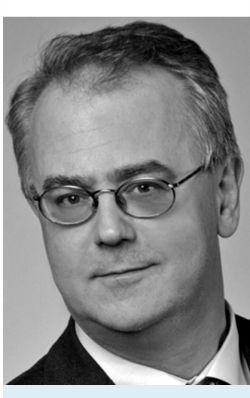

Johannes Wancata
Sozialpsychiatrie ist „jene Wissenschaft, die sich systematisch mit der Bedeutung von sozialen, kulturellen sowie Umgebungsfaktoren in weitestem Sinn für seelische Gesundheit und Krankheit befasst. Sie bezieht dabei soziologische, sozialpsychologische und kulturanthropologische Momente sowohl in Bezug auf die allgemeine Beeinflussung der Auffassungen von Gesundheit und Krankheit als auch deren Bedeutung für den einzelnen in ihre Betrachtung ein. Sie beschäftigt sich im Besonderen mit der Diagnose, Prognose, Therapie und Vorbeugung psychischer Krankheiten in und für Gruppen von Menschen“ [1]. Somit gehören auch die Beschäftigung mit jenen mikro- und makrosozialen Bedingungen, die zu Krankheit führen und die Kranken beeinflussen, die Nutzung dieser Aspekte in der Behandlung und die Beschäftigung mit epidemiologischen Fragen zu den zentralen Aufgaben der Sozialpsychiatrie [2-4]. Da die Beschäftigung mit dem sozialen, räumlichen und zeitlichen Kontext zu den Hauptaufgaben der Sozialpsychiatrie gehört, ist es nicht verwunderlich, dass die Bemühungen um die Rückkehr und Integration von schwer psychisch Kranken in die Gesellschaft einer der Schwerpunkte des Sozialpsychiatrie war und ist. Die Bemühungen um die Enthospitalisierung chronisch psychisch Kranker und um die Umstrukturierung der gemeindefernen psychiatrischen Großkrankenhäuser (sowie teilweise sogar deren Auflösung) hat die Sozialpsychiatrie in den letzten Jahrzehnten so stark geprägt, dass diese Bemühungen fast zu einem Synonym für Sozialpsychiatrie wurden. Dabei gelangen in den letzten 4 Jahrzehnten eindrucksvolle Leistungen: keinem anderen medizinischen Fachgebiet ist es gelungen, die Versorgungsstrukturen derart umzugestalten wie dies der Psychiatrie gelungen ist.

Die Berücksichtigung des sozialen, räumlichen und zeitlichen Kontextes ist aber natürlich nicht nur in Bezug auf die Versorgungseinrichtungen, sondern vor allem auch im klinischen Alltag von großer Bedeutung. Sozialpsychiatrisch-therapeutische Aktivitäten wie Zeitstrukturierung, die Arbeit mit dem sozialen Umfeld (vor allem mit den Angehörigen) oder die berufliche Rehabilitation sind für viele Kranke essenziell [5]. Ebenso ist es bedeutsam, soziale und andere Umgebungsfaktoren wie Migration, Arbeitslosigkeit oder Kultur auch in der Diagnostik und Therapieplanung zu berücksichtigen. Vieles was vor 4 Jahrzehnten aus Engagement und basierend auf „Bauchgefühl“ begonnen wurde, ist heute wissenschaftlich gut beforscht und wir wissen, welche Aspekte wir hier im klinischen Alltag berücksichtigen müssen. So wie in anderen Bereichen der Psychiatrie eine gewisse Spezialisierung (z.B. stationäre Akutbehandlung) hilfreich sein kann, kann es ebenso sinnvoll sein, dass sich manche auf die Behandlung von Menschen aus einem anderen Kulturkreis oder auf psychiatrische Rehabilitation spezialisieren. Insofern kann im klinischen Alltag eine Subspezialisierung auf einzelne Bereiche, die besonders hohes sozialpsychiatrisches Wissen erfordert, günstig sein. Ob derartige Spezialisierungen für die Versorgung der Kranken förderlich oder eher hinderlich sind, hängt aber von der lokalen Organisation und Struktur der psychiatrischen Versorgung ab.

Die Kranken haben ein Recht darauf, dass nicht nur bei der medikamentösen Behandlung, sondern auch bei sozialpsychiatrisch-therapeutischen Aktivitäten gut untersucht ist, was wirklich hilft und möglichst wenig „Nebenwirkungen“ hat. In ähnlicher Weise wollen politische Entscheidungsträger berechtigterweise basierend auf Evidenz wissen, welche Schritte zur Verbesserung der Versor- gungsstrukturen nötig sind [6-8]. Aber auch zahlreiche andere wissenschaftliche Fragen stehen dringend an, wie zum Beispiel: Wie können psychische Krankheiten frühzeitig erkannt werden? Wie sollen Kranke am besten mit der Erfahrung von Stigma umgehen? Was wäre nötig, um „Heavy Usern“ besser passende Angebote machen zu können? Sozialpsychiatrie benötigt also - genauso wie die biologische und psychotherapeutische Psychiatrie - Forschung!

Im Bereich der Forschung Schwerpunkte zu setzen ist wichtig, denn Experte wird nur, wer sich ausführlich mit einem Spezialgebiet auseinandersetzt. In der Forschung haben oft umschriebene und eng definierte Forschungsansätze Vorteile, wenn unter Kontrolle anderer potenzieller Einflüsse Hypothesen geprüft werden sollen. (Dabei darf aber auch nicht übersehen werden, dass der Vorteil der experimentellen Überschaubarkeit nicht zu einem eingeengten, reduktionistischen Bild psychischer Erkrankungen führen darf, wenn die Ergebnisse klinisch relevant sein sollen.)

Orte der Forschung sind überwiegend Universitäten - diese stehen heute aber unter einem enormen Erfolgsdruck. Erfolg an Universitäten meint, hohe Summen an Forschungsgeldern einzuwerben und Publikationen in möglichst renommierten Journalen zu veröffentlichen. Jede Abteilung oder Klinik muss daher vor allem jene fördern, die in diesen Aspekten erfolgreich sind. Innerhalb der Psychiatrie hat aber die biologisch-psychiatrische Forschung Vorteile, da neben der Industrie, die psychopharmakologische Forschung in großem Umfang fördert, auch manche akademische Fördergeber vorwiegend biologische Grundlagenforschung unterstützen. Die Tatsache, dass rein biologisch-psychiatrische Journale im Vergleich zu sozialpsychiatrischen Journalen häufig deutlich höher gerankt sind, ist eine altbekannte Tatsache [9], der in der Wissenschafts-Community auch dadurch Rechnung getragen wurde, 
dass es neben dem "Science Citation Index“ auch einen „Social Science Citation Index" gibt. Wenn Abteilungen nicht ganz klar und eindeutig sozialpsychiatrischen Fragestellungen gewidmet sind, wird sozialpsychiatrische Forschung für junge Wissenschaftler völlig uninteressant sein, weil sie sich gegen jene, die biologisch-psychiatrisch forschen, nur sehr schwer durchsetzen können. Wenn sich aber junge Wissenschaftler innerhalb des Rahmens einer sozialpsychiatrischen Universitätsabteilung untereinander messen, haben sie realistische Chancen - somit ist es auch attraktiv, sich mit sozialpsychiatrischen Fragestellungen zu beschäftigen.

Um das, was die Sozialpsychiatrie in den letzten Jahrzehnten entwickelt hat, auch weiterhin im klinischen Alltag anbieten zu können, ist es nötig, dass Medizinstudenten und junge Ärzte dies erlernen. Die Schwerpunkte in der studentischen Lehre werden aber häufig vor allem bei jenen Themen gesetzt, die auch beforscht werden. Insofern ist zu befürchten, dass ohne sozialpsychiatrische Forschung die Weitergabe des Wissens an die nächste Generation von Ärzten (aber auch anderer Berufsgruppen) leidet. Um sozialpsychiatrische Themen in der studentischen (aber auch der postgradualen) Lehre zu verankern, ist es also essenziell, entsprechend spezialisierte universitäre Einrichtungen zu etablieren.

Diese wenigen Aspekte zeigen nur beispielhaft, dass es vor allem im universitären Bereich spezialisierte und klar gewidmete sozialpsychiatrische Einrichtungen braucht. Ohne Einrichtungen der Forschung und Lehre, die explizit sozialpsychiatrischen Themen gewidmet sind, würde die Bedeutung der Sozialpsychiatrie in der Lehre und Forschung, aber in der Folge auch im klinischen Alltag an Bedeutung verlieren. Wenn wir die Behandlung und die Lebenssituation der Kranken verbessern wollen, wird es im klinischen Alltag aber unumgänglich sein, mit der biologischen und der psychotherapeutischen Psychiatrie integrativ zusammenzuarbeiten.

\section{Literatur}

1 Strotzka H. Einführung in die Sozialpsychiatrie. RoRoRo Studium 14. Reinbeck bei Hamburg: Rowohlt; 1972

2 Fleck S. Social psychiatry - an overview. Soc Psychiatry Psychiatr Epidemiol 1990; 25: $48-55$

3 Freedman DX. Psychiatric epidemiology counts. Arch Gen Psychiatry 1984; 41: 931-933

4 Tschiesner R, Schweigkofler H, Favaretto E et al. Zur Epidemiologie suizidalen Verhaltens: Eine Untersuchung im Gesundheitsbezirk Brixen. Neuropsychiatr 2012; 26: 121-128
5 Kreiner B, Baranyi A, Stepan A et al. Psychoedukation und Lebensqualität: Eine Erweiterung der Grazer Evaluationsstudie (Teil 1). Neuropsychiatr 2012; 26: 7-14

6 Lauber C. Krankenversorgung - Versorgungsplanung - Versorgungspolitik - Versorgungsforschung. Psychiat Prax 2012; 39: $1-2$

7 Frajo-Apor B, Macha I, Kemmler G et al. Mechanische Bewegungseinschränkungen: Die klinische Praxis an einer psychiatrischen Universitätsklinik. Neuropsychiatr 2013 27: $84-91$

8 Kirchen-Peters S, Fehrenbach R-A, Diefenbacher $A$. Brauchen Pflegeheime gerontopsychiatrische Liaisondienste? Ergebnisse einer Pilotstudie in fünf Pflegeheimen. Psychiat Prax 2012; 39: 14-20

9 Heidenreich $U$, Baethge $C$. Deutschsprachige psychiatrische Zeitschriften in der Bundesrepublik - eine quantitative Bestandsaufnahme. Psychiat Prax 2012; 39: 26-33

\section{Korrespondenzadresse}

Univ.-Prof. Dr. Johannes Wancata

Klinische Abt. für Sozialpsychiatrie,

Univ.-Klinik für Psychiatrie und Psychotherapie,

Medizinische Universität Wien

Währinger Gürtel 18-20

1090 Wien, Österreich

johannes.wancata@meduniwien.ac.at

\section{Bibliografie}

Dol http://dx.doi.org/

10.1055/s-0033-1349590

Psychiat Prax 2013; 40: 412-413

(c) Georg Thieme Verlag KG

Stuttgart · New York

ISSN 0303-4259 